\title{
Treatment of chronic viral hepatitis with alpha-interferon: A consensus conference report
}

THE CASL HePATITIS CONSENSUS GROUP

$\mathrm{C}_{\mathrm{h}}^{\mathrm{n}}$ HRONIC HEPATITIS DUE TO EITHER hepatitis B virus (HBV) or hepatitis $\mathrm{C}$ virus (HCV) is a serious and potentially fatal disease. Alpha-interferon was recently approved in Canada for the treatment of chronic HBV or HCV hepatitis and is the only approved therapeutic agent. This article provides clinical practice guidelines to assist practitioners and patients in making decisions about the indications and benefits of treatment with interferon for chronic viral hepatitis.

\section{DEVELOPMENT OF GUIDELINES}

The conference was held in Montreal November 26 to 27, 1993 under the auspices of the Canadian Association for the Study of the Liver and of the Canadian Liver Foundation. The participants were physicians with interest and experience in the management of patients with chronic viral hepatitis.
Before the conference, each participant answered a questionnaire dealing with indications for treatment, dosage, side effects and patient management. Results were analyzed and discussed the conference, and the guidelines presented in this paper are a representation of the opinion of these experts. They are intended as practical information for patient management and should be used in conjunction with specific product information and sound medical judgement. The guidelines are augmented with brief background information on interferon and on the natural history of chronic HBV and HCV hepatitis, and with selected key references.

\section{ALPHA-INTERFERON}

Interferons are a family of nonspecific regulatory proteins that inhibit the replication of several viruses. Alpha-interferon is produced by leukocytes and

Organizing chairman: Dr P-Michel Huet (President of the Canadian Association for the Study of the Liver [CASL]). Participants: Dr Paul Adams, Dr Frank Anderson, Dr Robert Bailey, Dr Vincent G Bain, Dr Victor Feinman, Dr Cameron N Ghent, Dr Jenny Heathcote, Dr Sam Lee, Dr Gary Levy, Dr Thomas I Michalak, Dr Gerald Minuk, Dr Khazal Paradis, Dr Eve Roberts, Dr Linda J Scully, Dr Eldon Shaffer, Dr Averell H Sherker, Dr Morris Sherman, Dr Mark Swain, Dr Jean-Pierre Villeneuve (chairman for hepatitis B), Dr Bernard Willems (chairman for hepatitis C), Dr Noël Williams, Dr Helga Witt-Sullivan

Correspondance and reprints: Dr Jean-Pierre Villeneuve or Dr Bernard Willems, André-Viallet Clinical Research Center, Hôpital Saint-Luc, 264, René-Lévesque est, Montréal, Québec H2X 1P1. Telephone (514) 281-2446, Fax (514) 281-2492

Received for publication April 1, 1994. Accepted April 10, 1994

This paper is also being published in The Canadian Journal of Infectious Diseases, Volume 5 Number 3, May/June 1994

beta-interferon by fibroblasts. Gammainterferon, produced by $\mathrm{T}$ lymphocytes, is a different class of molecules that is closer to cytokines than to alpha- and beta-interferons. Within the alpha class, at least 16 different subtypes are known. In addition to their effect on viral replication, interferons influence cell growth and differentiation and modulate the immune response.

Three types of alpha-interferon are commercially available in Canada: interferon-alpha-2a (Roferon, HoffmannLa Roche Ltd) and interferon-alpha-2b (Intron A, Schering Canada Inc) are genetically engineered (ie, recombinant interferons), whereas Wellferon (Burroughs Wellcome Inc) is produced by a lymphoblastoid cell line after induction by Sendai virus and contains multiple subtypes of alpha-interferon.

\section{THE NATURAL HISTORY OF CHRONIC HEPATITIS B}

The evolution of acute hepatitis B towards chronicity depends largely on the age at which the infection is acquired. More than $90 \%$ of infected neonates develop chronic hepatitis. When the disease is contracted in adulthood, approximately $5 \%$ go on to become chronic carriers.

The natural history of chronic hepatitis B has now been well described (1) and a representative example is shown in Figure 1. Chronic hepatitis B can be divided into two phases: an initial rep- 


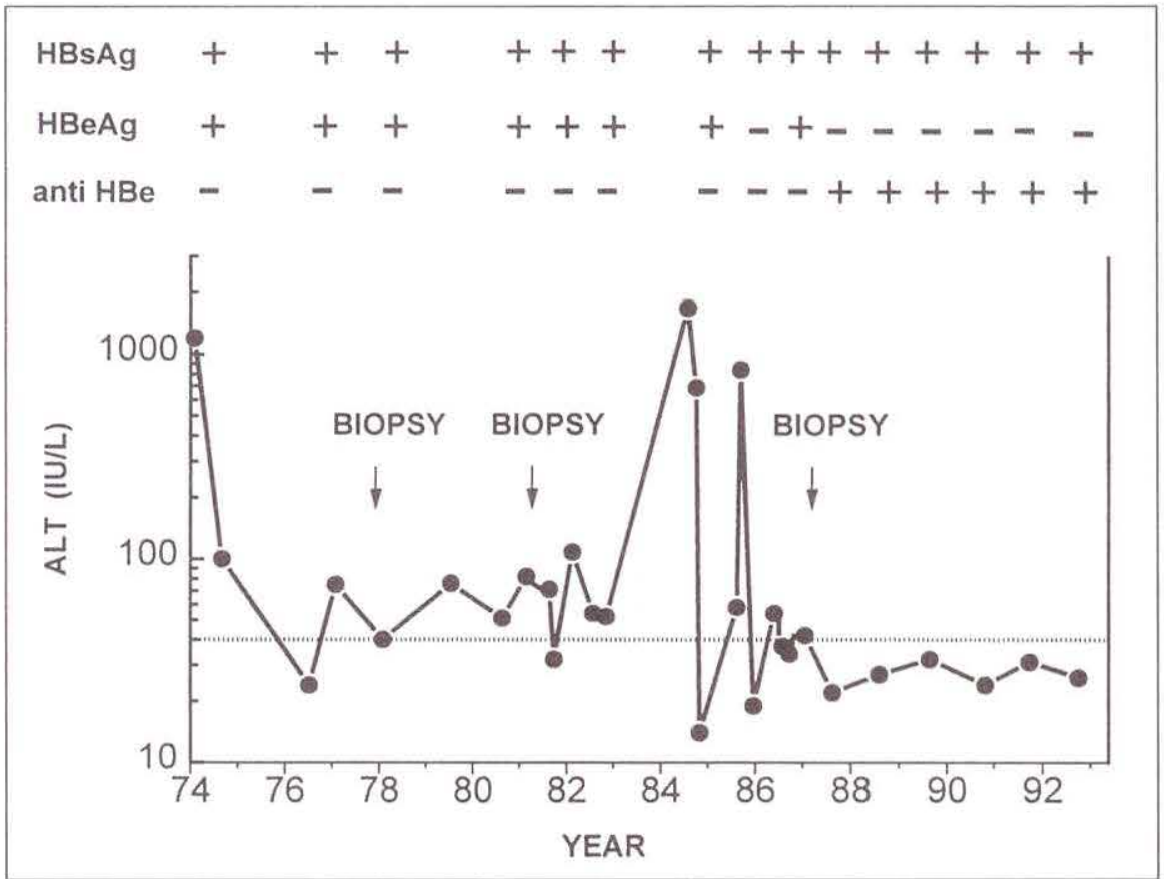

Figure 1) The natural history of chronic hepatitis B: a representative example. A 34-year-old man developed acute type $B$ hepatitis in 1974 and became a chronic carrier of the hepatitis B virus. From 1974 to 1985, there was evidence of active viral replication (positive hepatitis B e antigen [HBeAg] in serum and positive hepatitis B core antigen on liver biopsy). A first biopsy in 1977 showed chronic hepatitis and a second one in 1981 chronic hepatitis with fibrosis. In 1984-85, a sharp rise in serum alanine aminotransferase (ALT) was followed by a spontaneous seroconversion from $\mathrm{HBe} A \mathrm{~A}$-positive to anti-HBe-positive with subsequent normalization of serum transaminases. The patient became asymptomatic and a third biopsy in 1987 showed inactive cirrhosis. He remained hepatitis B surface antigen $(\mathrm{HBs} \mathrm{Ag})$-positive

licative phase and a later nonreplicative phase. During the initial phase there is evidence of ongoing viral replication, as indicated by the presence of hepatitis $\mathrm{B}$ e antigen $(\mathrm{HBeAg})$ and HBV. DNA in serum, and liver cell necrosis, as indicated by abnormal serum transaminases. This initial phase lasts for only a few years in some subjects, but may persist for decades in others. Some authors have further subdivided the replicative phase into an immune tolerance phase followed by an immune clearance phase to account for the observation that serum transaminases can be initially normal, particularly in carriers of Asian origin. Then at some stage in the natural history of the disease, viral replication stops or decreases with the disappearance of $\mathrm{HBeAg}$, appearance of anti-HBe antibodies and normalization of serum transaminases. HBV-DNA is no longer measurable by conventional hybridization assays, but can be detected in serum by sensitive molecular amplification techniques. It may therefore be more appropriate to describe this phase as low replicative or latent hepatitis B infection. Carriers remain hepatitis B surface antigen ( $\mathrm{HBs} \mathrm{Ag})$-positive, and about $1 \%$ of carriers who have entered the nonreplicative phase will lose $\mathrm{HBsAg}$ each year. According to the length and severity of the initial replicative phase, $\mathrm{HBsAg}$ carriers may have lesions ranging from near-normal liver to cirrhosis with the complications of portal hypertension (ascites, hepatic encephalopathy, variceal bleeding) and remain at risk for hepatocellular carcinoma.

\section{ALPHA-INTERFERON IN CHRONIC HEPATITIS B}

Indications for treatment: The purpose of treatment is to induce a transition from the replicative to the nonreplicative phase (ie, $\mathrm{HBeAg}$ to anti-HBe seroconversion), which will result in a reduction of hepatic inflammation and, consequently, liver damage. Since seroconversion may occur spontaneously, it is important to follow new patients for at least six months before considering any treatment to ensure that they are truly chronic carriers and that they are not clearing virus on their own.

There are now approximately 20 published randomized controlled trials that have examined the role of alphainterferon in the treatment of chronic hepatitis B. Review of these trials (2) and other published work allows rational recommendations for treatment to be formulated. The most suitable patients for interferon treatment have the following characteristics: first, positive $\mathrm{HBs} A g$ and $\mathrm{HBeAg}$ for at least six months; second, raised transaminases; third, no evidence of hepatic decompensation; fourth, white blood cell count greater than $4000 \times 10^{9}$ cells $/ \mathrm{L}$ (neutrophil count greater than $1500 \times 10^{9}$ cells $/ \mathrm{L}$ ) and platelets greater than $80 \times 10^{9}$ cells $/ \mathrm{L}$. With interferon therapy, 30 to $50 \%$ of these patients will successfully clear viral replication and their liver disease will become quiescent. These patients should be treated with interferon by an experienced physician providing no contraindications exist (see below).

A number of other patient categories warrant specific comments regarding interferon treatment:

- Normal liver enzymes: patients who are positive for $\mathrm{HBeAg}$ have active viral replication, but some patients have little or no active hepatic inflammation. This subgroup has not been well studied and treatment with interferon is not recommended since it is believed that they are unlikely to respond. Some have advocated a course of steroids before interferon treatment, however this is of unproven efficacy.

- Coinfection with hepatitis D virus: the hepatitis $D$ (delta) virus can exist only in the presence of the HBV. When present, it is usually associated with aggressive liver disease and rapid progression to cirrhosis and liver failure. Unfortunately, interferon treatment has been only of marginal benefit in this subgroup. Its use is associated with a high 
relapse rate and should only be undertaken by those with extensive experience with viral hepatitis.

\section{- Coinfection with HBV and} human immunodeficiency virus: coinfection with human immunodeficiency virus (HIV) identifies a subgroup with a poor response to interferon. Although a small number of patients may respond, the results with interferon are not substantially different from results with placebo, and treatment is not recommended. Pilot studies of combination treatment with interferon plus zidovudine also documented a poor response.

\section{- Coinfection with HBV and}

HCV: coinfection with these two hepatitis viruses may be seen because both are spread parenterally. There appears to be a reciprocal relationship between the replicative status of hepatitis B and hepatitis $\mathrm{C}$ in cases of coinfection. In patients with HBV dominance (positive $\mathrm{HBeAg}$ and HBV-DNA), the presence of hepatitis $\mathrm{C}$ does not preclude a response to interferon.

- Precore mutant: this HBV variant has a mutation in the precore region of the core gene. This results in an inability to synthesize $\mathrm{HBeAg}$; however, the virus retains its pathogenecity. These patients therefore have raised transaminases and active hepatic inflammation despite positivity for anti-HBe. The presence of a precore mutant is implied by positivity for HBV-DNA in serum and/or hepatitis B core antigen staining within hepatocytes on liver biopsy. Response rates to interferon ranging from 20 to $50 \%$ have been demonstrated in European trials, however relapse appears to be more common than in native HBV infections. Treatment in this subset of patients is deserving of further study.

- Decompensated cirrhosis, $\mathrm{HBeAg}$ positive: whereas interferon is well tolerated in cirrhotic patients with only rare evidence of hepatic decompensation, even low dose interferon in decompensated patients has been associated with significant side effects, including bacterial infections, gastrointestinal bleeding and deteriorating hepatic function. Limited data in this patient subgroup show benefit in some patients, but there have also been fatalities associated with interferon treatment. Interferon cannot be generally recommended in these patients.

- Chronic hepatitis B associated glomerulonephritis: renal injury in these patients is mediated by immune complexes made up of hepatitis $B$ viral antigens and host antibodies. Pilot studies have shown that response to treatment results in marked decreases in proteinuria as well as correction of hypoalbuminemia in patients who are $\mathrm{HBeAg}$-positive and have elevated serum transaminases. Patients with renal histology showing membranous glomerulonephritis are highly responsive to interferon, whereas those showing membranoproliferative glomerulonophritis are unlikely to have a persistent response.

\section{- Hepatitis B recurrence postliver} transplantation: patients with end-stage liver disease secondary to chronic hepatitis B infection do poorly as a group after liver transplantation because of a high rate of recurrence in the new engrafted liver. These patients are difficult to treat because of their long term need for immunosuppression and a very high viral load. Experience with interferon in these patients has been

disappointing and treatment is not recommended.

- Chronic hepatitis B in children: there have been few studies of alpha-interferon therapy in children, and the role of interferon in treating children is not well established. These patients should be referred to tertiary care centres.

Treatment - dosage regimen and monitoring response: There is no con- clusive evidence to indicate a significant difference in the efficacy of different alpha-interferons (Intron A, Roferon and Wellferon). The recommended dose for treating adult chronic $\mathrm{HBV}$ patients who are $\mathrm{HBeAg}$ - and HBVDNA-positive is $10 \times 10^{6} \mathrm{U}$ subcutaneously three times weekly or $5 \times 10^{6} \mathrm{U}$ daily to a total of no more than $35 \times 10^{6} \mathrm{U}$ per week. Duration of treatment should be 16 weeks. The use of other agents in combination with alpha-interferon is not recommended.

A loss of HBeAg and the appearance of anti-HBe are the goals of treatment with interferon. The loss of HBeAg from the serum is often associated with an improvement in symptoms, enzyme abnormalities, HBV-DNA levels and histology; infectivity is also decreased. In 90 to $95 \%$ of patients who respond to interferon therapy, $\mathrm{HBeAg}$ seroconversion (from positive to negative) occurs within six months of completion of treatment. The presence of $\mathrm{HBeAg}$ in the serum six months following discontinuation of interferon therapy suggests treatment failure.

In addition to confirming the diagnosis of HBV infection in difficult cases, serum HBV-DNA levels can be used as a marker of the extent of viral replication. Recent data indicate that quantitation of HBeAg can provide similar information. In the context of documenting the effect of interferon therapy on HBV viral replication, monitoring HBV-DNA levels is desirable but not mandatory.

A pretreatment biopsy is preferable but not mandatory in patients with chronic type B hepatitis. A liver biopsy performed before interferon therapy is useful in helping to identify patients who are likely to respond to treatment (those with active histological disease). It can also identify previously undiagnosed, coexisting disorders such as hemochromatosis, fatty liver, etc. However, the indications and contraindications for interferon treatment are based on clinical, biochemical and serological findings and not on histology.

Post-treatment liver biopsies are not recommended outside of clinical trials. The goal of interferon therapy for chronic HBV infection (HBeAg serocon- 
version) is identified by serological testing.

Failure and relapse: Failure of alphainterferon therapy is defined as persistence of HBeAg and HBV-DNA in serum and failure of serum transaminases to normalize. No additional treatment with interferon is indicated in patients in whom treatment failed. There are no data in the literature to indicate that prolonged treatment, higher doses or another brand of interferon is likely to enhance the response rate.

A relapse is defined as reappearance of HBV-DNA and HBeAg with elevation of serum transaminases in a patient who has previously responded to alphainterferon therapy. Patients who relapse merit being retreated with interferon. It is acknowledged that there are no data in the literature, either for or against therapy, to provide a guide, but given a good response initially it is likely that the patient will respond again. The duration of the second course is unknown, but should probably not be less than four months.

\section{THE NATURAL HISTORY OF HEPATITIS C}

The majority of patients who contract HCV will remain chronically infected and at least $50 \%$ will develop chronic hepatitis. Among chronic carriers of $\mathrm{HCV}$, a small percentage may have normal transaminases and very mild liver disease. However, chronic hepatitis $\mathrm{C}$ will lead to cirrhosis in at least $20 \%$ of cases and to liver failure in $5 \%$. The progression of post-transfusion hepatitis $\mathrm{C}$ to hepatocellular carcinoma is well documented. Hence, the evolution, severity and time course of the liver disease induced by $\mathrm{HCV}$ are unpredictable, but may be severe (3).

A diagnosis of chronic hepatitis $\mathrm{C}$ is suggested by the presence of abnormal alanine aminotransferase (ALT) for at least six months, with anti-HCV positivity. The transaminase level usually fluctuates in a 'yo-yo' pattern. Consequently, one cannot easily conclude that the disease progression has stopped. Since screening tests for antiHCV may be falsely positive in autoimmune chronic hepatitis (lupoid hepatitis), this diagnosis should be ex- cluded by adequate clinical and laboratory evaluation before interferon treatment is considered.

\section{ALPHA-INTERFERON IN CHRONIC HEPATITIS C}

Alpha-interferon has been shown to normalize transaminases in approximately $40 \%$ of treated patients (4). However, when the treatment is stopped, half of those who have responded will relapse. It is hoped, but yet not proven, that among treated patients, at least those with a complete and sustained response (approximately $20 \%$ of treated patients) will have an improvement in the natural history of their disease.

Indications for treatment: Given the spontaneous fluctuations of ALT and $\mathrm{HCV}$ viremia and our limited knowledge on the natural history of chronic hepatitis $\mathrm{C}$, a rational approach to treatment indications and end-points is still lacking. However, the patient most likely to respond to interferon therapy and to benefit from it would fulfil all these criteria: first, anti-HCV positivity, with ALT levels at least twice normal for at least six months; second, chronic hepatitis or compensated cirrhosis (ChildPugh score less than 10); third, neutrophil count greater than $1500 \times 10^{9}$ cells/L; and fourth, no evidence of autoimmune disease.

Patients with hemophilia, thalassemia or essential mixed cryoglobulinemia who satisfy the above criteria can also be considered for treatment, as well as those with $\mathrm{HBs} A g$ positivity who are in the nonreplicative phase of the disease (for those who have chronic hepatitis B and active HBV replication, see recommendation in hepatitis $B$ section).

Hepatitis C-positive patients with decompensated cirrhosis (Child-Pugh greater than 10) may be treated in some circumstances, but the practitioner should proceed with caution, and it is recommended this type of patient be treated only in tertiary referral centres. These patients may also be considered for liver transplantation.

Interferon treatment is not recommended if any of these criteria are met: first, anti-HIV positive patients; second, active illicit drug use or alcohol abuse; third, persistently normal transaminase activity; fourth, evidence of autoimmune hepatitis, because severe acute exacerbation of autoimmune hepatitis with liver failure may occur if interferon is administered.

A liver biopsy is highly recommended before initiation of treatment for all patients with no contraindication to this procedure. A post-treatment biopsy is not routinely necessary. Working definitions of the response to interferon treatment: The efficacy of interferon treatment is assessed from repeated measurements of serum transaminases. The measurement of HCV. RNA by any technique, either qualitatively or quantitatively, is not routinely necessary before or during treatment. Long term disappearance of HCV-RNA may turn out to be a marker of efficacy. However, the relationship of HCV-RNA status to clinically important outcomes remains to be demonstrated.

According to the pattern of ALT (and of HCV-RNA, if available), the response to interferon treatment can be classified into four types:

- Complete sustained response: a return to normal level of ALT and disappearance of HCV-RNA during the therapy, with maintenance of normal ALT levels and negative HCV-RNA for at least six months after stopping therapy.

- Complete response with relapse: a return to normal level of ALT and disappearance of HCV-RNA during therapy, with development of abnormal ALT levels or reappearance of HCV-RNA within six months of stopping therapy.

- Breakthrough: a return of ALT levels to normal and disappearance of HCV-RNA during therapy, with a subsequent rise to abnormal levels of ALT or reappearance of HCV-RNA while still on therapy.

- Nonresponse: failure to achieve normalization of ALT levels. A nonresponse status after eight weeks of therapy constitutes an appropriate time to consider escalation of dosage.

The aim of the treatment is to induce a complete and sustained re- 
sponse. All other outcomes constitute treatment failure. There are no data to indicate whether treatment is useful to patients having a decrease in ALT levels during treatment, but without a complete sustained response.

Treatment - dosage regimen and monitoring response: The recommended regimen for treatment of chronic HCV infection is to start with interferon $3 \times 10^{6} \mathrm{U}$ three times weekly. If the ALT normalizes at this dose, then the treatment should be continued for a total of 24 weeks at $3 \times 10^{6} \mathrm{U}$ thrice weekly. If the ALT does not normalize by eight weeks, then the dose of interferon should be increased to $5 \times 10^{6} \mathrm{U}$ three times weekly. If the ALT normalizes after four weeks at this dose, then the interferon should be continued at $5 \times 10^{6} \mathrm{U}$ for a total duration of treatment of 24 weeks. If the ALT does not normalize after a total of 12 weeks with the increase in dosage, the interferon should be stopped.

If the patient relapses after an initial response to treatment, retreatment is not routinely recommended. Retreatment could be considered in patients with very severe liver disease or associated conditions such as vasculitis, but each case should be considered separately. At present, maintenance therapy with interferon is not routinely recommended. Both conditions are being evaluated in prospective trials.

There is no conclusive evidence to indicate significant differences in the efficacy of different alpha-interferons (Intron A, Roferon and Wellferon).

Interferon treatment should be initiated and monitored by specialists or practitioners with experience in the treatement of hepatitis. ALT measurement is the keystone of the monitoring. Given interferon-related side effects and complications (5), an adequate follow-up is recommended during and after treatment. In case of complete sustained response, ALT should be measured at six, 12, 18 and 24 months, and yearly thereafter for up to five years. The relapse rate after fulfilling the definition of complete sustained response is believed to be low, but data on long term follow-up are lacking.

\section{COMPLICATIONS OF INTERFERON THERAPY}

Neuropsychiatric: Neuropsychiatric complications of interferon therapy occur in 10 to $20 \%$ of patients, and are more common in the elderly and in patients with cirrhosis. Typically they appear one to three months following initiation of therapy. Although a past medical history or positive family history of affective disorders does not increase the risk of interferon-induced neuropsychiatric complaints, a previous history of central nervous system insults may be associated with more severe side effects, such as delirium and suicidal tendencies. It is important to note that many patients with chronic viral hepatitis may complain of fatigue and depression as a result of their infection, and interferon therapy in these individuals may improve these symptoms. Interferon therapy should be withheld in patients with ongoing neuropsychiatric symptoms and those with a history of previous central nervous system insults or injury. Unless the indications for treatment are strong, interferon should also be withheld in patients with a past history of psychosis. If neuropsychiatric symptoms appear during interferon therapy, the dose should be decreased by $50 \%$ unless the symptoms are severe, in which case the drug should be discontinued immediately and arrangements made for the patient to be assessed by a psychiatrist.

Neutropenia: A $40 \%$ decline in absolute neutrophil counts should be expected in patients starting interferon therapy. The maximum fall in neutrophil counts occurs early (day 4). A more marked decline can be expected in patients with underlying portal hypertension. Interferon therapy should be avoided in patients with neutrophil counts less than $1500 \times 10^{9}$ cells/L before therapy. If the neutrophil count falls below $1000 \times 10^{9}$ cells/L during therapy, the dosage of interferon should be decreased by $50 \%$ and discontinued if an improvement is not apparent within one to two weeks.

Thrombocytopenia: A 20\% fall in platelet counts should be expected in patients starting interferon therapy.
The fall is rapid (maximum on day 2), and a more marked decline can be expected in patients with underlying portal hypertension. Interferon therapy should be avoided in patients with platelet counts less than $80 \times 10^{9} / \mathrm{L}$ before therapy. If the platelet count falls below $50 \times 10^{9} / \mathrm{L}$ during therapy, the dosage should be reduced by $50 \%$ and discontinued if an improvement is not apparent within one to two weeks. It is important to note that patients with interferon-induced thrombocytopenia often have coexisting neutropenia.

Autoimmune disorders: Patients on interferon therapy may develop autoantibodies, usually two to three months post-initiation of therapy. Although the interferon-induced autoantibodies resolve within 16 months of discontinuing treatment, interferon-induced autoimmune disorders may persist despite discontinuation of therapy. Interferon can also exacerbate preexisting autoimmune disorders. Patients with active autoimmune diseases, particularly autoimmune chronic active hepatitis (lupoid hepatitis), should not be treated with interferon. Interferon should be discontinued if previously inactive autoimmune disease becomes active or signs of autoimmune disease appear during therapy. In particular, autoimmune hyperthyroidism and hypothyroidism may appear during therapy or even after interferon has been stopped, and adequate monitoring is recommended.

Renal dysfunction: The most common renal side effect of interferon therapy is proteinuria, occuring in 15 to $20 \%$ of treated patients. Despite renal clearance of interferon, increased toxicity and altered pharmacokinetics are not a feature of interferon treatment in patients with chronic renal failure. Interferon therapy need not be withheld in patients with chronic renal failure. The appearance of mild, asymptomatic proteinuria is not an indication for altering the dose of interferon. If renal disorders other than mild proteinuria occur during therapy the interferon should be discontinued.

Diabetes: Preexisting diabetes, particularly if mild or well controlled, is not 
considered a contraindication to the use of interferon. However, glucose levels should be monitored carefully if interferon is initiated in a diabetic patient. If control becomes difficult, interferon therapy should be stopped. Severe or brittle preexisting diabetes is felt to be a relative contraindication to the use of interferon therapy. If diabetes develops during a course of interferon therapy, it is recommended that treatment be discontinued.

Cardiac failure: Interferon should be used cautiously in patients with a history of cardiovascular disease. In patients with preexisting mild or well compensated heart failure, one could initiate therapy, however cardiac status should be monitored closely throughout therapy. If heart failure, to whatever degree, occurs de novo during therapy, it is recommended that interferon be discontinued.

Pulmonary infiltrates: Respiratory complications, albeit rare, have been described with interferon use, particularly at higher doses. If a patient develops new respiratory symptoms or a significant worsening of an underlying respiratory problem with the addition of new pulmonary infiltrates on chest $\mathrm{x}$ ray, it is recommended that interferon be discontinued.

ACKNOWLEDGEMENTS: The consensus conference was held under the auspices of the CASL and the Canadian Liver Foundation (CLF), and was supported by educational grants from Burroughs-Wellcome Inc, Hoffmann-La Roche Ltd and Schering Canada Inc.

\section{REFERENCES}

1. Sherlock S. The natural history of hepatitis B. Postgrad Med J 1987;63:7-11.

2. Wong DKH, Cheung AM, O'Rourke K, Naylor CD, Detsky AS, Heathcote J. Effect of alpha-interferon treatment in patients with hepatitis B e antigenpositive chronic hepatitis B. A meta-analysis. Ann Intern Med 1993;119:312-23.

3. Alberti A, Realdi G. Parenterallyacquired non- $A$, non- $B$ (type $C$ ) hepatitis. In: McIntyre N, Benhamou JP, Bircher J, Rizetto M, Rodes J, eds. Oxford Textbook of Clinical Hepatology. Oxford: Oxford Medical Publication, 1991:605-17.

4. Davis GL, Balart LA, Schiff ER, et al. Treatment of chronic hepatitis $\mathrm{C}$ with recombinant interferon alpha. $\mathrm{A}$ multicenter randomized, controlled trial. N Engl J Med 1989;321:1501-6.

5. Renault PF, Hoofnagle JH. Side effects of alpha interferon. Semin Liver Dis 1989;9:273-8. 


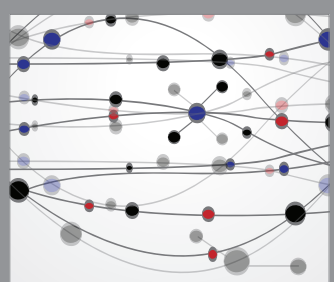

The Scientific World Journal
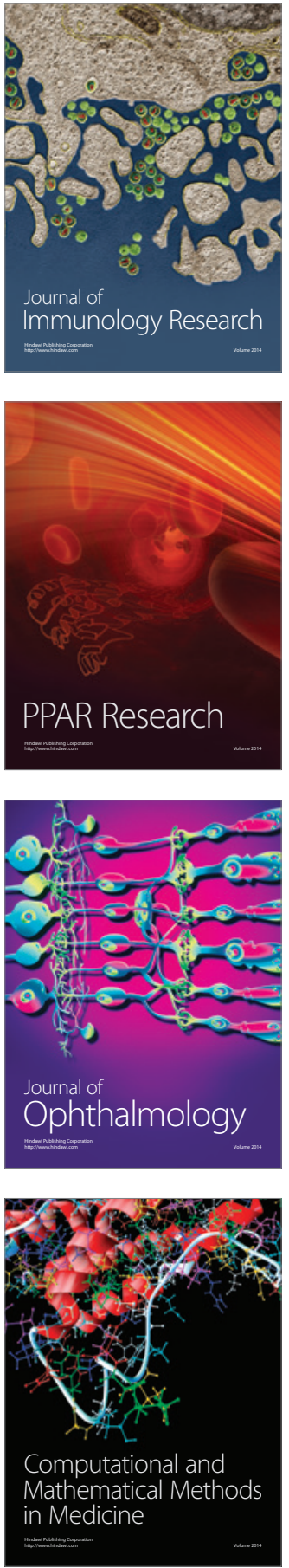

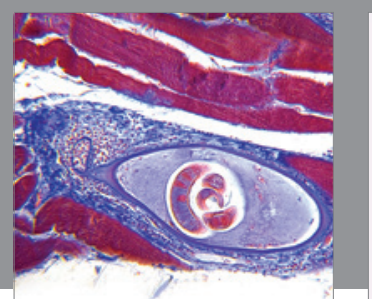

Gastroenterology Research and Practice

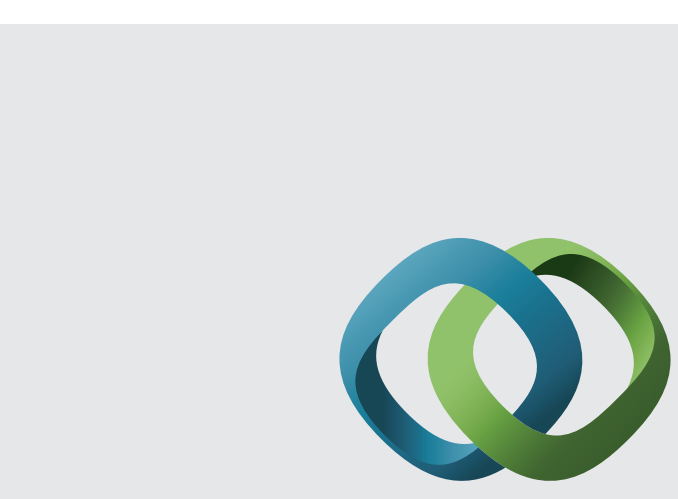

\section{Hindawi}

Submit your manuscripts at

http://www.hindawi.com
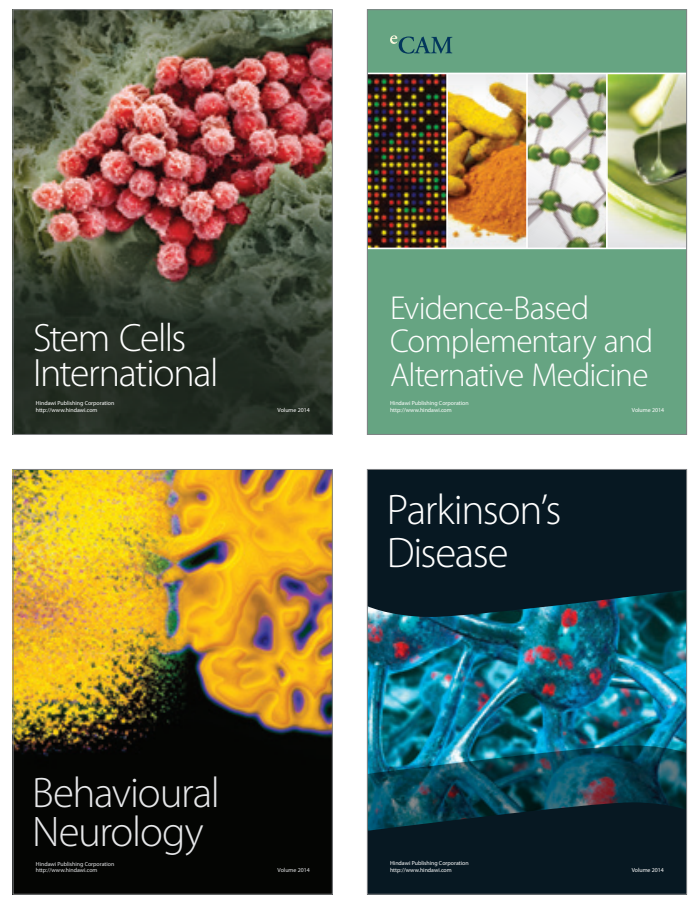
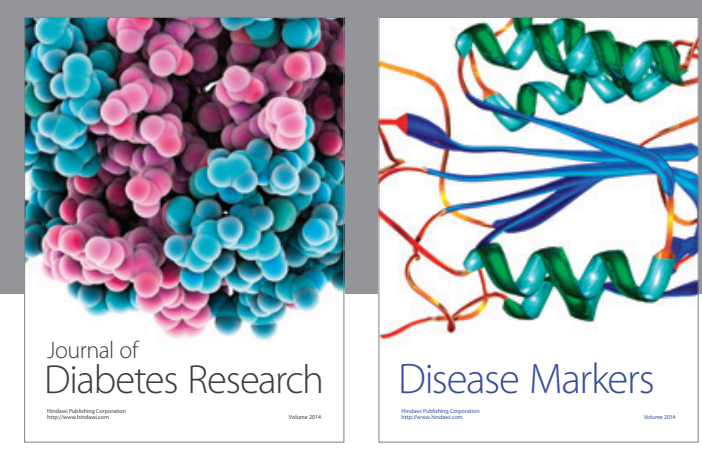

Disease Markers
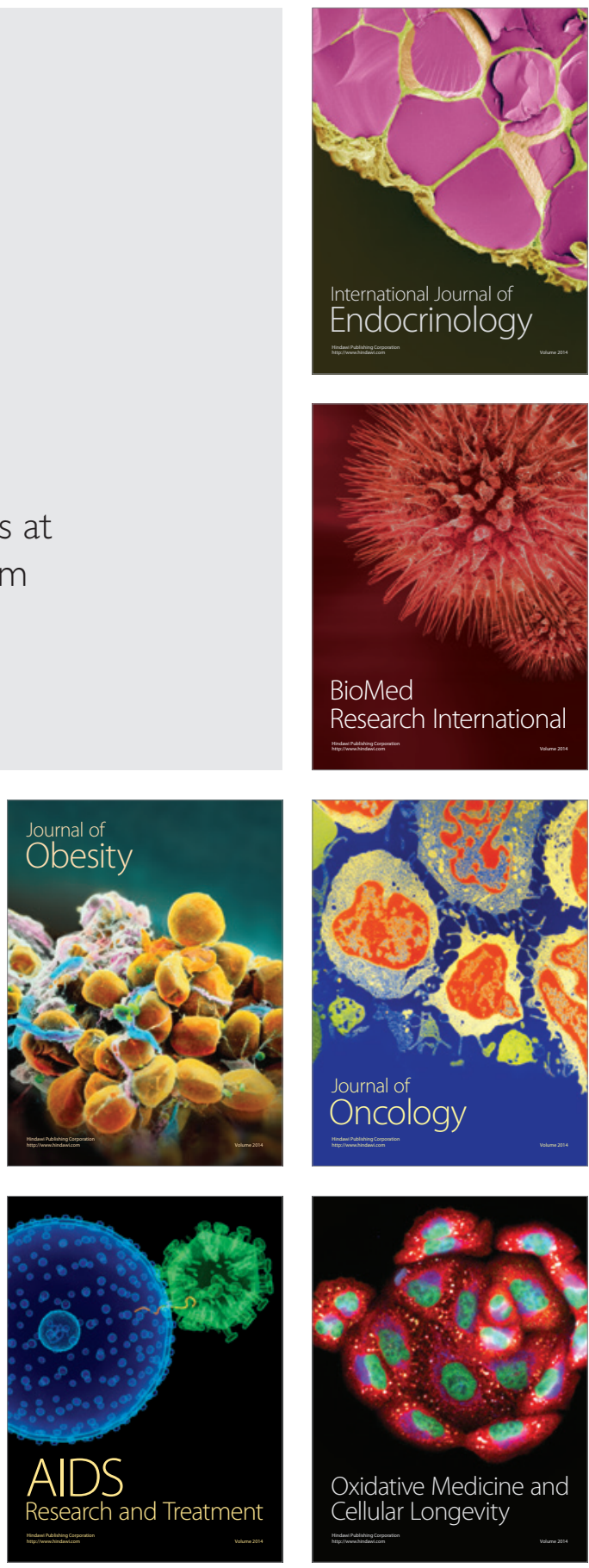